\title{
Correlates of non-target vessel-related adverse events in patients with ST-segment elevation myocardial infarction: insights from five-year follow-up of the EXAMINATION trial
}

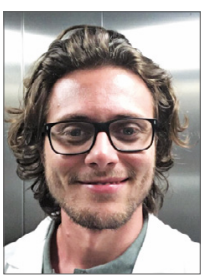

Giosafat Spitaleri $^{1}$, MD; Elisabetta Moscarella ${ }^{1}, \mathrm{MD}$; Salvatore Brugaletta ${ }^{*}, \mathrm{MD}, \mathrm{PhD}$;

Alberto Pernigotti ${ }^{1}, \mathrm{MD}$; Luis Ortega-Paz ${ }^{1}, \mathrm{MD}$; Josep Gomez-Lara ${ }^{2}$, MD, PhD;

Angel Cequier ${ }^{2}, \mathrm{MD}$; Andrés Iñiguez ${ }^{3}, \mathrm{MD}$; Antonio Serra ${ }^{4}, \mathrm{MD}$; Pilar Jiménez-Quevedo ${ }^{5}, \mathrm{MD}$;

Vicente Mainar $^{6}$, MD; Gianluca Campo ${ }^{7}$, MD; Maurizio Tespili ${ }^{8}$, MD; Peter den Heijer ${ }^{9}, \mathrm{MD}$;

Armando Bethencourt ${ }^{10}, \mathrm{MD}$; Nicolás Vazquez ${ }^{11}, \mathrm{MD}$; Marco Valgimigli ${ }^{12}, \mathrm{MD}, \mathrm{PhD}$;

Patrick W. Serruys ${ }^{13}$, MD, PhD; Manel Sabaté 1 , MD, PhD

1. University Hospital Clínic, Cardiovascular Clinic Institute, Institut d'Investigacions Biomèdiques August Pi i Sunyer (IDIBAPS), Barcelona, Spain; 2. Department of Cardiology, University Hospital of Bellvitge, Barcelona, Spain; 3. Department of Cardiology, Hospital do Meixoeiro, Vigo, Spain; 4. Department of Cardiology, University Hospital of Sant Pau, Barcelona, Spain; 5. Department of Cardiology, University Hospital San Carlos, Madrid, Spain; 6. Department of Cardiology, Hospital General of Alicante, Alicante, Spain; 7. Cardiovascular Institute, Azienda Ospedaliero-Universitaria di Ferrara, Cona (FE), Italy and Maria Cecilia Hospital, GVM Care \& Research, E.S. Health Science Foundation, Cotignola, Italy; 8. Department of Cardiology, University Hospital Bolognini Seriate, Bergamo, Italy; 9. Department of Cardiology, Amphia Ziekenhuis, Breda, the Netherlands; 10. Department of Cardiology, Hospital Son Espases, Palma de Mallorca, Spain; 11. Department of Cardiology, Hospital Juan Canalejo, A Coruña, Spain; 12. University Hospital of Bern, Inselspital, Bern, Switzerland; 13. International Centre of Circulatory Health, Imperial College London, London, United Kingdom

GUEST EDITORS: Lorenz Räber, MD, PhD; University Hospital Bern, Bern, Switzerland, and Alec Vahanian, MD, PhD; Department of Cardiology, Hôpital Bichat, Paris and University Paris VII, Paris, France.

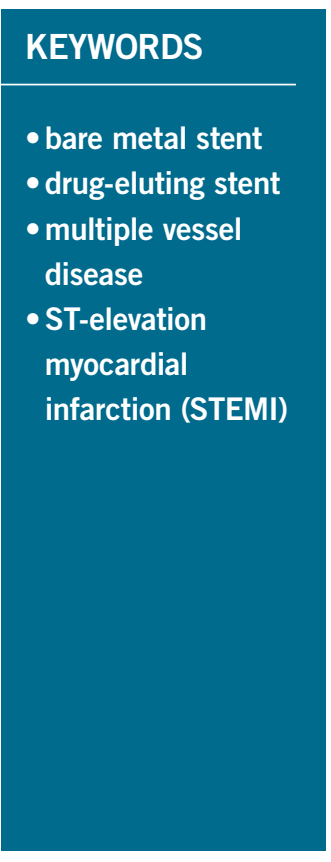

\section{Abstract}

Aims: The aim of this substudy was to determine the five-year correlates of non-TV-related adverse events (AE) in STEMI patients included in the EXAMINATION trial.

Methods and results: The EXAMINATION trial randomised 1,498 STEMI patients to bare metal or everolimus-eluting stent implantation. In this substudy, patients were analysed according to non-TV-related $\mathrm{AE}$, defined as the composite of either non-TV revascularisation (non-TVR) or non-TV-related myocardial infarction (MI). At five-year follow-up, 125 patients (8.3\%) exhibited 136 non-TV-related AE (124 [8.3\%] non-TVR, 12 [0.8\%] non-TV-related MI), accounting for $47.1 \%$ of 289 non-fatal cardiac events overall. These patients had a higher incidence of diabetes mellitus ( $\mathrm{p}<0.001)$, arterial hypertension $(\mathrm{p}=0.032)$, previous MI ( $\mathrm{p}=0.073)$, multivessel disease $(\mathrm{p}<0.001)$, and incomplete revascularisation $(\mathrm{p}=0.049)$, and a lower rate of ST-segment resolution $>70 \%(\mathrm{p}=0.042)$ as compared to the rest. At Cox analysis, previous MI (HR 1.872, 95\% CI: 1.004-3.489; $\mathrm{p}=0.048$ ), incomplete revascularisation (HR 1.746, 95\% CI: 1.029-2.963; $\mathrm{p}=0.039$ ) and diabetes (HR 1.942, 95\% CI: 1.292-2.919; $\mathrm{p}=0.001$ ) were independent correlates of non-TVrelated AE.

Conclusions: In STEMI patients undergoing primary percutaneous coronary intervention, previous MI, incomplete revascularisation and diabetes resulted in being independent correlates of five-year non-TVrelated AE.

\footnotetext{
*Corresponding author: Hospital Clínic, Cardiovascular Clinic Institute, Institut d'Investigacions Biomèdiques August Pi i Sunyer (IDIBAPS), Carrer Villarroel 170,08036 Barcelona, Spain.E-mail: sabrugal@clinic.ub.es
} 


Abbreviations
ACS
ARC
CABG
CAD
DES
IR
MACE
MI
MVD
Non-TV
Non-TV-related AE
Non-TVR
PCI
STEMI

\section{Introduction}

Drug-eluting stent (DES) trials focus mainly on device performance in terms of target lesion events ${ }^{1}$. Conversely, data on events related to non-target vessels (non-TV) are limited, especially in a specific population such as ST-elevation myocardial infarction $(\mathrm{STEMI})^{2}$. In patients with acute coronary syndrome (ACS) and multivessel disease (MVD) who underwent culprit only percutaneous coronary intervention (PCI), it has been demonstrated that $50 \%$ of total cardiac events are related to non-TV at three-year follow-up ${ }^{3}$. Although complete revascularisation can reduce the risk of major adverse cardiovascular events (MACE) ${ }^{4}$ in these patients, non-TV-related adverse events (AE) still account for almost a half of cardiac events ${ }^{5}$, strengthening the concept of coronary atherosclerosis as a diffuse and progressive disease that goes beyond the treatment of a single coronary lesion.

We therefore sought to analyse the incidence and correlates of non-TV-related AE at five-year follow-up in the STEMI patients included in the EXAMINATION trial ${ }^{6}$.

\section{Editorial, see page 1869}

\section{Methods}

\section{STUDY POPULATION}

This is a post hoc analysis from the all-comers, multicentre, controlled, and randomised, EXAMINATION trial (A Clinical Evaluation of Everolimus Eluting Coronary Stents in the Treatment of Patients With ST-segment Elevation Acute Myocardial Infarction: EXAMINATION Study) (NCT00828087). The detailed study design and five-year results of the EXAMINATION trial have been reported previously ${ }^{6,7}$. The EXAMINATION trial randomised 1:1 a total of 1,498 STEMI patients to an everolimus-eluting stent $(n=751)\left(X_{E N C E}{ }^{\circledR}\right.$; Abbott Vascular, Santa Clara, CA, USA) or a MULTI-LINK VISION ${ }^{\circledR}$ BMS (n=747) (Abbott Vascular). All participating centres submitted and received the approval of their medical ethics committee for the protocol and for the informed consent. The study was conducted in compliance with the protocol, the Declaration of Helsinki, and applicable local requirements. All patients provided written informed consent.

\section{ENDPOINTS AND DEFINITIONS}

All the patients were analysed according to the incidence of nonTV-related AE, defined as the composite of non-TV revascularisation (non-TVR) or non-TV-related myocardial infarction (MI), as per the Academic Research Consortium (ARC) definitions ${ }^{8}$. Because death cannot be assigned to any vessel segment, it was not counted as a TV or non-TV-related event. Incomplete revascularisation (IR) was defined as a residual stenosis of $\geq 50 \%$ in the left main coronary artery or $\geq 70 \%$ in another major epicardial coronary artery on the basis of operator visual estimation. Staged procedures were all performed within the first month after discharge and with the same stent as per randomisation. Therefore, incomplete revascularisation was evaluated after staged procedures were performed. Any revascularisation carried out after this time was counted as an unplanned intervention per protocol. Definitions of death (all-cause and cardiac) and stent thrombosis have already been reported ${ }^{7,9}$. All clinical events were adjudicated by an independent clinical events committee (Cardialysis, Rotterdam, the Netherlands).

\section{STATISTICAL ANALYSIS}

Continuous variables were expressed as mean \pm standard deviation or median and interquartile range, according to their distribution, and were compared by ANOVA or Wilcoxon rank-sum test. Categorical variables were expressed as numbers and percentages and were compared by chi-square analysis or Fisher's exact test, as appropriate.

In order to analyse independent correlates of five-year non-TVrelated AE, baseline clinical, procedural, and angiographic characteristics were tested in a univariable analysis. Those variables with $\mathrm{p}<0.10$ at the univariable analysis were then entered into a Cox multivariable model with backward elimination. MVD and staged procedure were not entered into the Cox model in order to avoid any multicollinearity with incomplete revascularisation, which conversely was included. Any relation between non-TV-related $\mathrm{AE}$ and TV-related AE, all-cause mortality and cardiac death was examined using a Cox regression model with non-TV-related AE as a time-varying covariate. In patients experiencing both a TV and a non-TV-related AE, we considered only those TV events that occurred after a non-TV-related AE.

Results from Cox regression analyses were reported as hazard ratios (HR) with associated 95\% confidence intervals and p-values. A two-tailed p-value $<0.05$ was considered to be statistically significant. Statistical analyses were performed using SPSS, Version 20.0 (IBM Corp., Armonk, NY, USA).

\section{Results POPULATION}

Complete five-year clinical follow-up was available in $97 \%$ of patients. At five-year follow-up, 125 patients (8.3\%) exhibited 136 non-TV-related AE (124 [8.3\%] non-TVR, 12 [0.8\%] non-TVrelated MI), accounting for $47.1 \%$ of 289 non-fatal cardiac events overall (Figure 1). Table 1 shows baseline clinical characteristics. 


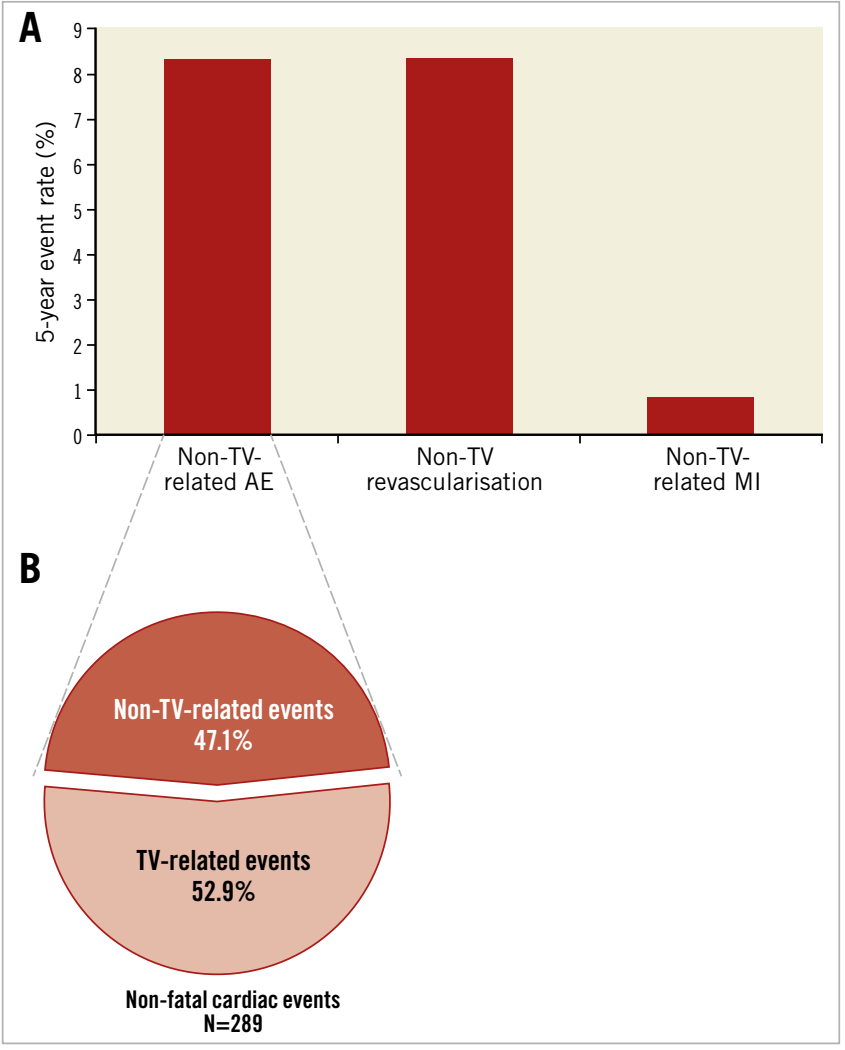

Figure 1. Non-TV-related adverse events. A) Incidence of non- $T V$ related adverse events, non-TV revascularisation and non-TV-related myocardial infarction. B) Proportion of non-TV-related adverse events of total non-fatal cardiac events. MI: myocardial infarction; Non-TV-related AE: non-target vessel-related adverse events; $T V$ : target vessel

Patients in the non-TV-related AE group had a higher incidence of diabetes mellitus $(p<0.001)$, arterial hypertension $(p=0.032)$, previous myocardial infarction $(p=0.073)$, MVD $(p<0.001)$, staged procedure $(\mathrm{p}<0.001)$, and incomplete revascularisation $(\mathrm{p}=0.049)$, and a lower rate of ST-segment resolution $>70 \%(\mathrm{p}=0.042)$ as compared to the other group. No other differences were found between the groups (Table 1, Table 2).

\section{CORRELATES OF NON-TARGET VESSEL-RELATED ADVERSE EVENTS}

At Cox analysis, previous MI (HR 1.872, 95\% CI: 1.004-3.489, $\mathrm{p}=0.048$ ), incomplete revascularisation (HR 1.746, 95\% CI: 1.029-2.963, $\mathrm{p}=0.039)$ and diabetes (HR 1.942, 95\% CI: $1.292-$ $2.919, \mathrm{p}=0.001)$ were independent correlates of non-TV-related AE (Figure 2).

Patients who experienced a non-TV-related AE had a higher risk of target vessel-related AE (HR 2.919, 95\% CI: 1.556-5.478, $\mathrm{p}<0.001$ ) (Table 3), even after adjustment (adjusted HR 2.818, 95\% CI: 1.470-5.404, $\mathrm{p}=0.002$ ), with no significant effect modification by type of stent implanted (HR for BMS: $2.011,95 \% \mathrm{CI}$ : 0.765-5.285; HR for DES: 3.095, 95\% CI: 1.625-9.676; $\mathrm{p}$ for interaction: 0.222 ). Non-TV events did not have a significant impact on cardiac death (HR 0.752, 95\% CI: 0.274-2.059, $\mathrm{p}=0.579$ ) or all-cause mortality (HR 0.891, 95\% CI: 0.435-1.825, p=0.753). Similarly, TV-related AE did not increase the risk of cardiac death (HR $0.891,95 \%$ CI: $0.435-1.825, \mathrm{p}=0.753$ ) or all-cause mortality (HR 1.357, 95\% CI: 0.624-2.950, $\mathrm{p}=0.442$ ).

In addition, median time from index procedure to non-TV events (median: 113 days; interquartile range: 44-604 days) and to TV events (median: 196 days; interquartile range: 27.5-678 days) was not statistically different $(p=0.96)$.

\section{Discussion}

Our analysis showed that: 1) the cumulative incidence of nonTV-related adverse events in STEMI patients treated with primary PCI was $8.3 \%$ at five years, accounting for $47.1 \%$ of total adverse events; 2) previous MI, incomplete revascularisation, and diabetes appeared to be independent correlates of non-TV-related AE in our study population; 3) non-TV-related adverse events are associated with a higher incidence of TV-related adverse events.

Coronary artery disease (CAD) progression involves the entire coronary tree and could be responsible for events in areas remote from the culprit vessel. Previous studies have already reported the rate of this phenomenon in various populations. Cutlip et al found that, in a low-risk population, the non-TVR cumulative rate was $21.7 \%$ at five-year follow up ${ }^{10}$. In the SIRIUS trial ${ }^{11}$, events

\begin{tabular}{|c|c|c|c|c|c|}
\hline \multicolumn{4}{|l|}{ Predictors of non-TV-related AE } & \multirow{2}{*}{$\begin{array}{c}\text { HR (95\% Cl) } \\
1.942(1.292-2.919)\end{array}$} & \multirow{2}{*}{$\begin{array}{c}p \text {-value } \\
0.001\end{array}$} \\
\hline Diabetes & & 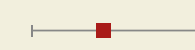 & & & \\
\hline Previous MI & & $\square$ & & $1.872(1.004-3.489)$ & 0.048 \\
\hline Incomplete revascularisation & & $\square$ & & $1.746(1.029-2.963)$ & 0.039 \\
\hline Hypertension & & $\square$ & & $1.192(0.820-1.733)$ & 0.358 \\
\hline \multirow[t]{2}{*}{ ST-segment resolution $\geq 70 \%$} & $1 \div$ & & & $0.738(0.510-1.068)$ & 0.107 \\
\hline & 0 & $\begin{array}{c}2 \\
\text { Hazard ratio }\end{array}$ & 3 & & \\
\hline
\end{tabular}

Figure 2. Correlates of non-TV-related AE at five-year follow-up. AE: adverse events; CI: confidence interval; HR: hazard ratio; MI: myocardial infarction; non-TV: non-target vessel 
Table 1. Baseline clinical characteristics.

\begin{tabular}{|c|c|c|c|c|c|}
\hline & $\begin{array}{l}\text { Patients with non-TV- } \\
\text { related events } N=125\end{array}$ & $\begin{array}{l}\text { Patients without non-TV- } \\
\text { related events } N=1,373\end{array}$ & Hazard ratio (95\% CI) & $p$-value \\
\hline \multicolumn{2}{|c|}{ Age, years $($ mean $\pm S D)$} & $62.45 \pm 11.61$ & $61.09 \pm 12.46$ & $1.009(0.995-1.023)$ & 0.210 \\
\hline \multicolumn{2}{|l|}{ Male gender, n (\%) } & $108(86.4)$ & $1,136(82.7)$ & $1.309(0.785-2.183)$ & 0.302 \\
\hline \multicolumn{2}{|l|}{ DES, n (\%) } & $63(50.4)$ & $688(50.1)$ & $1.005(0.708-1.427)$ & 0.977 \\
\hline \multirow{5}{*}{$\begin{array}{l}\text { Coronary risk } \\
\text { factors, n (\%) }\end{array}$} & Smoker & $89(71.2)$ & $993(72.4)$ & $0.955(0.779-1.170)$ & 0.654 \\
\hline & Diabetes mellitus & $37(29.6)$ & $221(16.1)$ & $2.112(1.438-3.101)$ & $<0.001$ \\
\hline & Arterial hypertension & $72(57.6)$ & $653(47.6)$ & $1.474(1.034-2.102)$ & 0.032 \\
\hline & Hyperlipidaemia & $47(37.6)$ & $608(44.3)$ & $0.769(0.536-1.105)$ & 0.156 \\
\hline & Family history & $22(17.6)$ & 231 (16.9) & $0.941(0.694-1.275)$ & 0.695 \\
\hline \multirow{5}{*}{$\begin{array}{l}\text { Cardiovascular } \\
\text { history, n (\%) }\end{array}$} & Previous MI & $11(8.9)$ & $69(5.0)$ & $1.768(0.952-3.282)$ & 0.071 \\
\hline & Previous $\mathrm{PCl}$ & $6(4.8)$ & $55(4.0)$ & $1.172(0.516-2.662)$ & 0.704 \\
\hline & Previous CABG & $0(0.0)$ & $10(0.7)$ & - & - \\
\hline & Previous stroke & $4(3.2)$ & $27(2.0)$ & $1.592(0.588-4.310)$ & 0.360 \\
\hline & Pre-infarction angina & $34(27.2)$ & $400(29.2)$ & $0.908(0.612-1.346)$ & 0.631 \\
\hline \multirow{4}{*}{$\begin{array}{l}\text { Clinical status } \\
\text { on admission, } \\
n(\%)\end{array}$} & Killip I & $111(88.8)$ & $1,226(89.6)$ & $0.943(0.541-1.644)$ & 0.836 \\
\hline & Killip II & $9(7.2)$ & $106(7.7)$ & $0.937(0.476-1.846)$ & 0.851 \\
\hline & Killip III & $3(2.4)$ & $20(1.5)$ & $1.609(0.512-5.059)$ & 0.416 \\
\hline & Killip IV & $2(1.6)$ & $16(1.2)$ & $1.422(0.352-5.751)$ & 0.621 \\
\hline \multirow{5}{*}{$\begin{array}{l}\text { Antiplatelet } \\
\text { regimen, n (\%) }\end{array}$} & Aspirin before $\mathrm{PCl}$ & $117(93.6)$ & $1,271(92.6)$ & $1.175(0.574-2.404)$ & 0.660 \\
\hline & Clopidogrel before $\mathrm{PCl}$ & $116(92.8)$ & $1,302(94.8)$ & $0.730(0.370-1.438)$ & 0.363 \\
\hline & GP IIb/IIla inhibitor & $48(38.4)$ & 521 (37.9) & $1.024(0.714-1.469)$ & 0.896 \\
\hline & Aspirin 1 year & $112(92.6)$ & $1,207(89.5)$ & $1.454(0.738-2.868)$ & 0.280 \\
\hline & Clopidogrel 1 year & $109(92.4)$ & $1,157(89.1)$ & $1.466(0.743-2.893)$ & 0.270 \\
\hline \multirow{3}{*}{$\begin{array}{l}\text { Cardiac markers, } \\
\text { median } \\
\text { [interquartile } \\
\text { range] }\end{array}$} & CK peak & $1,342[553-2,702]$ & $1,435[654-2,939]$ & $1.000(0.999-1.000)$ & 0.717 \\
\hline & CK-MB peak & 162 [71-284] & 147 [60-329] & $1.000(0.986-1.000)$ & 0.831 \\
\hline & Troponin peak & $17[4-78]$ & $17[4-70]$ & $1.000(0.992-1.002)$ & 0.611 \\
\hline \multicolumn{2}{|c|}{ Multivessel disease, $\mathrm{n}(\%)$} & $48(38.4)$ & $299(21.8)$ & $2.185(1.523-3.133)$ & $<0.001$ \\
\hline \multicolumn{2}{|c|}{ Staged procedure, n (\%) } & $32(25.6)$ & $198(14.4)$ & $1.991(1.332-2.975)$ & $<0.001$ \\
\hline \multicolumn{2}{|c|}{ Incomplete revascularisation, $\mathrm{n}(\%)$} & $17(13.6)$ & $115(8.4)$ & $1.705(1.022-2.843)$ & 0.041 \\
\hline
\end{tabular}

Table 2. Procedural characteristics.

\begin{tabular}{|c|c|c|c|c|c|}
\hline & $\begin{array}{l}\text { Patients with non-TV- } \\
\text { related events } N=125\end{array}$ & $\begin{array}{l}\text { Patients without non-TV- } \\
\text { related events } N=1,373\end{array}$ & Hazard ratio $(95 \%$ CI $)$ & $p$-value \\
\hline \multirow{5}{*}{$\begin{array}{l}\text { Infarct-related } \\
\text { artery, n (\%) }\end{array}$} & Left main & $0(0.0)$ & $4(0.3)$ & $0.000(0.000-2.173)$ & 0.959 \\
\hline & Left anterior descending artery & $49(39.2)$ & $555(40.5)$ & $0.883(0.605-1.289)$ & 0.520 \\
\hline & Left circumflex & $12(9.6)$ & $136(9.9)$ & $0.842(0.484-1.464)$ & 0.542 \\
\hline & Right coronary artery & $59(47.2)$ & $594(43.3)$ & $1.540(0.213-11.121)$ & 0.668 \\
\hline & Saphenous vein graft & $1(0.8)$ & $4(0.3)$ & $2.342(0.449-12.214)$ & 0.397 \\
\hline \multicolumn{2}{|c|}{ TIMI flow 0 before $\mathrm{PCI}, \mathrm{n}(\%)$} & $70(56.0)$ & $808(59.2)$ & $0.900(0.632-1.281)$ & 0.557 \\
\hline \multicolumn{2}{|c|}{ Number of stents, mean \pm SD } & $1.39 \pm 0.66$ & $1.38 \pm 0.65$ & $1.007(0.768-1.321)$ & 0.958 \\
\hline \multicolumn{2}{|c|}{ Total stent length, mm, mean \pm SD } & $27.91 \pm 15.42$ & $27.48 \pm 13.87$ & $1.002(0.990-1.015)$ & 0.706 \\
\hline \multicolumn{2}{|c|}{ Post-dilation, n (\%) } & $22(17.6)$ & 199 (14.5) & $1.243(0.784-1.969)$ & 0.355 \\
\hline \multicolumn{2}{|c|}{ ST-segment resolution $>70 \%, \mathrm{n}(\%)$} & $63(54.3)$ & $789(63.8)$ & $0.682(0.473-0.982)$ & 0.040 \\
\hline \multirow{3}{*}{$\begin{array}{l}\text { Anticoagulant } \\
\text { regimen, } \\
n(\%)\end{array}$} & Unfractionated heparin & $102(81.6)$ & $1,087(79.2)$ & $1.170(0.744-1.840)$ & 0.496 \\
\hline & Low molecular weight heparin & $15(12.0)$ & $118(8.6)$ & $1.395(0.814-2.393)$ & 0.226 \\
\hline & Bivalirudin & $10(8.0)$ & $95(6.9)$ & $1.159(0.607-2.211)$ & 0.655 \\
\hline
\end{tabular}


Table 3. Impact of non-TV-related adverse events on clinical outcomes from Cox regression model with non-TV-related adverse events as a time-varying covariate.

\begin{tabular}{|l|c|r|}
\hline & Hazard ratio (95\% CI) & p-value \\
\hline TV-related adverse events & $2.818(1.470-5.404){ }^{*}$ & 0.002 \\
\hline TV revascularisation & $3.228(1.748-5.926)$ & $<0.001$ \\
\hline TV-related MI & $1.546(0.361-6.608)$ & 0.557 \\
\hline All-cause death & $1.357(0.624-2.950)$ & 0.442 \\
\hline Cardiac death & $0.891(0.435-1.825)$ & 0.753 \\
\hline $\begin{array}{l}\text { Definite, probable or possible } \\
\text { stent thrombosis }\end{array}$ & $0.198(0.028-1.427)$ & 0.108 \\
\hline $\begin{array}{l}\text { Definite or probable stent } \\
\text { thrombosis }\end{array}$ & 1.927 (0.444-8.361) & 0.381 \\
\hline $\begin{array}{l}\text { *Adjusted hazard ratio. Non-TV-related adverse events defined as the } \\
\text { composite of non-TV revascularisation and non-TV-related MI. TV-related } \\
\text { adverse events defined as the composite of TV revascularisation and } \\
\text { TV-related MI. Cl: confidence interval; MI: myocardial infarction; } \\
\text { TV: target vessel }\end{array}$ \\
\hline
\end{tabular}

attributed to non-TV were estimated at $26 \%$ after five years from the index procedure. Similarly, a single-centre all-comers Japanese study showed that the rate of non-TV repeat PCI was $17.6 \%$ at five-year follow-up ${ }^{12}$. Compared to these studies, we report a lower rate of events in the non-culprit vessels, which accounted for almost $50 \%$ of cardiac events overall. This lower rate of events could be explained in several ways. Firstly, the aforementioned findings might have been influenced by protocol-driven repeat coronary angiographies, whereas the events in our analysis were clinically driven. In addition, patients included in the previous studies had a higher CAD burden. In fact, MVD was present in up to $70 \%$ of those patients while in our population the prevalence of MVD was $30.1 \%$. This could also be related to the fact that we included only STEMI patients, who usually have a lower prevalence of MVD compared with other ACS patients ${ }^{13}$. In our population, the incidence of non-TV-related revascularisation was not statistically different between patients receiving bare metal stents and those receiving DES ( $8.3 \%$ vs. $8.3 \%, \mathrm{p}=0.975)$, even when considering patients who underwent a staged procedure in non-TV ( $15.8 \%$ vs. $11.6 \%, \mathrm{p}=0.353)$.

With regard to prognostic factors, in the present report we found that previous MI, incomplete revascularisation, and diabetes appeared to be independent factors of non-TV-related AE. It is already known that STEMI patients who have had a previous MI have worse long-term clinical outcomes ${ }^{14}$. Our findings are consistent with previous studies as we showed that previous MI is associated with an increased risk of non-TV-related AE. This could be explained by considering that STEMI patients who have experienced a previous MI could have a more extensive and complex CAD. Regarding the completeness of coronary revascularisation, it has been demonstrated that MVD, which is found in approximately $40-60 \%$ of STEMI patients, is associated with bad short- and long-term prognosis ${ }^{15}$. Several studies have analysed the effects of complete revascularisation on outcomes, but contrasting results have been reported. A recently published meta-analysis of eight studies showed that, in STEMI patients, complete revascularisation reduced the risk of MACE and repeat revascularisation, compared to incomplete revascularisation ${ }^{16}$. Two recent randomised controlled trials have shown that the fractional flow reserve-guided treatment of non-culprit vessels in STEMI patients reduced MACE at 12- to 24-month follow-up ${ }^{4,17}$. However, these studies examined MACE without giving information on non-TVrelated adverse events. Our study, which has a longer follow-up (60 months), focused on these events and showed that incomplete revascularisation is an independent risk factor for non-TVrelated AE. In addition, we found that, out of 17 non-TV-related AE occurring in patients with incomplete revascularisation, 13 were clinically driven non-TV revascularisations and that they occurred in the first three months after PCI. Therefore, we might hypothesise that these events could be related to lesions that were not treated immediately and that had an early impact on patients' symptoms, confirming the protective role of the complete revascularisation even in the first months after PCI.

Diabetes is an established risk factor for CAD and future cardiac events in patients who undergo PCI, mainly because of an increased risk of stent restenosis and MI during follow-up ${ }^{18}$. However, diabetes also plays a central role in the progression of atherosclerotic disease and it has been recognised as a negative prognostic factor for non-TV events. An intravascular coronary ultrasound analysis which included 237 coronary segments from 45 patients enrolled in the DIABETES I, II and III trials ${ }^{19-21}$ showed that insulin-dependent diabetes was associated with vessel shrinkage and disease progression, both in treated and in not previously treated lesions ${ }^{22}$. In the PROSPECT trial, patients with insulin-requiring diabetes had a threefold increased risk of nonculprit lesion-related MACE at three-year follow-up ${ }^{3}$. In addition, a recent propensity-matched sub-analysis of the PROSPECT trial showed that the three-year total MACE rate was significantly higher in patients with diabetes versus those without, mainly driven by a higher rate of non-culprit lesion-related $\mathrm{MACE}^{23}$. Accordingly, in our study population diabetes was the strongest predictor with almost a twofold risk of non-TV-related AE at fiveyear follow-up. This slightly lower risk of adverse events, as compared to the PROSPECT trial, could be related to the fact that we included all diabetic patients, regardless of insulin or oral glucoselowering medication use.

Interestingly, our analysis also showed that non-TV-related AE, despite the lack of a significant impact on all-cause mortality and cardiac death, are associated with a higher incidence of TV-related AE, in particular TVR. In addition, no statistically significant difference was found in the occurrence of any stent thrombosis between the two groups. The strong relation between non-TV and TV-related AE is a novel finding, which confirms the concept of $\mathrm{CAD}$ as an extensive disease. Therefore, our findings underline the fact that both complete revascularisation and an aggressive control of all cardiovascular risk factors (in particular diabetes) could be effective in the prevention of non-TV-related AE at longterm follow-up. 


\section{Limitations}

This study has several limitations. Firstly, it is a post hoc analysis and therefore our results should be considered hypothesis-generating. Secondly, there were baseline differences between both groups in terms of patient, clinical, lesion, and procedural characteristics; despite these differences, an adequate statistical treatment was performed in order to minimise their impact on the results. Neither prasugrel nor ticagrelor had been approved for use in clinical practice at the time this study was conducted, thus all patients were treated with clopidogrel. Moreover, we took into account only anatomical incomplete revascularisation rather than the functional residual CAD.

Although non-TV-related AE did not increase the risk of more prognostically relevant events such as death or myocardial infarction, $95 \%$ confidence intervals were wide, suggesting imprecision and uncertainty on the assessed relationship.

Lastly, patients with previous revascularisation (with either PCI or coronary artery bypass graft $[\mathrm{CABG}]$ ) were not excluded from the analysis; therefore, a non-TV AE could relate to a stent or graft failure rather than to a disease progression. However, only six patients, out of the 125 patients who experienced a non-TV adverse event, had undergone a previous PCI and none of them had undergone a previous CABG. This small number of patients should not have affected the results.

\section{Conclusions}

In STEMI patients undergoing primary PCI, incomplete revascularisation, previous $\mathrm{MI}$ and diabetes resulted in being independent correlates of five-year non-TV-related AE.

\section{Impact on daily practice}

Our findings might help in the identification, at the time of primary PCI, of those patients at risk of future events related to areas remote from the target vessel. Complete revascularisation at the index procedure and more aggressive risk factor control, in particular diabetes, could reduce the occurrence of non-target vessel-related adverse events at long-term follow-up.

\section{Guest Editor}

This paper was guest edited by Lorenz Räber, MD, PhD; University Hospital Bern, Bern, Switzerland, and Alec Vahanian, MD, PhD; Department of Cardiology, Hôpital Bichat, Paris, and University Paris VII, Paris, France.

\section{Conflict of interest statement}

The authors have no conflicts of interest to declare. The Guest Editor Lorenz Räber has no conflicts of interest to declare. The Guest Editor Alec Vahanian is a consultant for Edwards Lifesciences.

\section{References}

1. Byrne RA, Joner M, Kastrati A. Stent thrombosis and restenosis: what have we learned and where are we going? The Andreas Grüntzig Lecture ESC 2014. Eur Heart J. 2015;36:3320-31.
2. Colombo A, Latib A. Disease progression is as important as culprit lesion treatment. J Am Coll Cardiol. 2012;59:800-1.

3. Stone GW, Maehara A, Lansky AJ, de Bruyne B, Cristea E, Mintz GS, Mehran R, McPherson J, Farhat N, Marso SP, Parise H, Templin B, White R, Zhang Z, Serruys PW; PROSPECT Investigators. A prospective natural-history study of coronary atherosclerosis. N Engl J Med. 2011;364:226-35.

4. Smits PC, Abdel-Wahab M, Neumann FJ, Boxma-de Klerk BM, Lunde K, Schotborgh CE, Piroth Z, Horak D, Wlodarczak A, Ong PJ, Hambrecht R, Angerås O, Richardt G, Omerovic E; Compare-Acute Investigators. Fractional Flow Reserve-Guided Multivessel Angioplasty in Myocardial Infarction. N Engl J Med. 2017;376:1234-44.

5. Zellweger MJ, Kaiser C, Jeger R, Brunner-La Rocca HP, Buser P, Bader F, Mueller-Brand J, Pfisterer M. Coronary artery disease progression late after successful stent implantation. $J \mathrm{Am}$ Coll Cardiol. 2012;59:793-9.

6. Sabaté M, Brugaletta S, Cequier A, Iñiguez A, Serra A, Jiménez-Quevedo P, Mainar V, Campo G, Tespili M, den Heijer P, Bethencourt A, Vazquez N, van Es GA, Backx B, Valgimigli M, Serruys PW. Clinical outcomes in patients with ST-segment elevation myocardial infarction treated with everolimus-eluting stents versus bare-metal stents (EXAMINATION): 5-year results of a randomised trial. Lancet. 2016;387:357-66.

7. Sabaté M, Cequier A, Iñiguez A, Serra A, HernándezAntolín R, Mainar V, Valgimigli M, Tespili M, den Heijer P, Bethencourt A, Vázquez N, Brugaletta S, Backx B, Serruys P. Rationale and design of the EXAMINATION trial: a randomised comparison between everolimus-eluting stents and cobalt-chromium bare-metal stents in ST-elevation myocardial infarction. EuroIntervention. 2011;7:977-84.

8. Cutlip DE, Windecker S, Mehran R, Boam A, Cohen DJ, van Es GA, Steg PG, Morel MA, Mauri L, Vranckx P, McFadden E, Lansky A, Hamon M, Krucoff MW, Serruys PW; Academic Research Consortium. Clinical end points in coronary stent trials: a case for standardized definitions. Circulation. 2007;115: 2344-51.

9. Sabate M, Cequier A, Iñiguez A, Serra A, HernandezAntolin R, Mainar V, Valgimigli M, Tespili M, den Heijer P, Bethencourt A, Vazquez N, Gómez-Hospital JA, Baz JA, MartinYuste V, van Geuns RJ, Alfonso F, Bordes P, Tebaldi M, Masotti M, Silvestro A, Backx B, Brugaletta S, van Es GA, Serruys PW. Everolimus-eluting stent versus bare-metal stent in ST-segment elevation myocardial infarction (EXAMINATION): 1 year results of a randomised controlled trial. Lancet. 2012;380: 1482-90.

10. Cutlip DE, Chhabra AG, Baim DS, Chauhan MS, Marulkar S, Massaro J, Bakhai A, Cohen DJ, Kuntz RE, Ho KK. Beyond restenosis: five-year clinical outcomes from second-generation coronary stent trials. Circulation. 2004;110:1226-30.

11. Chacko R, Mulhearn M, Novack V, Novack L, Mauri L, Cohen SA, Moses J, Leon MB, Cutlip DE. Impact of target lesion and nontarget lesion cardiac events on 5-year clinical outcomes 
after sirolimus-eluting or bare-metal stenting. JACC Cardiovasc Interv. 2009;2:498-503.

12. Kaneko H, Yajima J, Oikawa Y, Tanaka S, Fukamachi D, Suzuki S, Sagara K, Otsuka T, Matsuno S, Kano H, Uejima T, Koike A, Nagashima K, Kirigaya H, Sawada H, Aizawa T, Yamashita T. Long-term incidence and prognostic factors of the progression of new coronary lesions in Japanese coronary artery disease patients after percutaneous coronary intervention. Heart Vessels. 2014;29:437-42.

13. André R, Bongard V, Elosua R, Kirchberger I, Farmakis D, Häkkinen U, Fusco D, Torre M, Garel P, Araújo C, Meisinger C, Lekakis J, Malmivaara A, Dovali M, Pereira M, Marrugat J, Ferrières J. International differences in acute coronary syndrome patients' baseline characteristics, clinical management and outcomes in Western Europe: the EURHOBOP study. Heart. 2014;100: 1201-7.

14. Puymirat E, Aissaoui N, Lemesle G, Cottin Y, Coste P, Schiele F, Ferrières J, Simon T, Danchin; FAST-MI Investigators. Long-Term Clinical Outcomes According to Previous Manifestations of Atherosclerotic Disease (from the FAST-MI 2010 Registry). Am J Cardiol. 2017;119:692-7.

15. Park DW, Clare RM, Schulte PJ, Pieper KS, Shaw LK, Califf RM, Ohman EM, Van de Werf F, Hirji S, Harrington RA, Armstrong PW, Granger CB, Jeong MH, Patel MR. Extent, location, and clinical significance of non-infarct-related coronary artery disease among patients with ST-elevation myocardial infarction. JAMA. 2014;312:2019-27.

16. Wang $\mathrm{CH}$, Zhang SY, Jin XF. Complete revascularization versus culprit-only revascularization in ST-segment elevation myocardial infarction and multivessel disease patients undergoing primary percutaneous coronary intervention: A meta-analysis and trial sequential analysis. Int J Cardiol. 2017;228:844-52.

17. Engstrøm T, Kelbæk H, Helqvist S, Høfsten DE, Kløvgaard L, Holmvang L, Jørgensen E, Pedersen F, Saunamäki K, Clemmensen P, De Backer O, Ravkilde J, Tilsted HH, Villadsen AB, Aarøe J, Jensen SE, Raungaard B, Køber L; DANAMI-3-PRIMULTI Investigators. Complete revascularisation versus treatment of the culprit lesion only in patients with ST-segment elevation myocardial infarction and multivessel disease (DANAMI-3 - PRIMULTI): an open-label, randomised controlled trial. Lancet. 2015;386: $665-71$.
18. Farkouh ME, Domanski M, Sleeper LA, Siami FS, Dangas G, Mack M, Yang M, Cohen DJ, Rosenberg Y, Solomon SD, Desai AS, Gersh BJ, Magnuson EA, Lansky A, Boineau R, Weinberger J, Ramanathan K, Sousa JE, Rankin J, Bhargava B, Buse J, Hueb W, Smith CR, Muratov V, Bansilal S, King S 3rd, Bertrand M, Fuster V; FREEDOM Trial Investigators. Strategies for multivessel revascularization in patients with diabetes. $N$ Engl $\mathrm{J} \mathrm{Med.} \mathrm{2012;367:}$ 2375-84.

19. Sabaté M, Jiménez-Quevedo P, Angiolillo DJ, GómezHospital JA, Alfonso F, Hernández-Antolín R, Goicolea J, Bañuelos C, Escaned J, Moreno R, Fernández C, FernándezAvilés F, Macaya C; DIABETES Investigators. Randomized comparison of sirolimus-eluting stent versus standard stent for percutaneous coronary revascularization in diabetic patients: the Diabetes and Sirolimus-eluting Stent (DIABETES) Trial. Circulation. 2005;112:2175-83.

20. Jiménez-Quevedo P, Sabaté M, Angiolillo DJ, Alfonso F, Hernández-Antolín R, SanMartín M, Gómez-Hospital JA, Bañuelos C, Escaned J, Moreno R, Fernández C, FernándezAvilés F, Macaya C; DIABETES Investigators. Long-term clinical benefit of sirolimus-eluting stent implantation in diabetic patients with de novo coronary stenoses: long-term results of the DIABETES trial. Eur Heart J. 2007;28:1946-52.

21. Jiménez-Quevedo P, Hernando L, Gómez-Hospital JA, Iñiguez A, SanRoman A, Alfonso F, Hernández-Antolín R, Angiolillo DJ, Bañuelos C, Escaned J, Gonzalo N, Fernández C, Macaya C, Sabaté M; DIABETES Investigators. Sirolimus-eluting stent versus bare metal stent in diabetic patients: the final five-year follow-up of the DIABETES trial. EuroIntervention. 2013;9: 328-35.

22. Jiménez-Quevedo $P$, Suzuki $N$, Corros $C$, Ferrer $C$, Angiolillo DJ, Alfonso F, Hernández-Antolín R, Bañuelos C, Escaned J, Fernández C, Costa M, Macaya C, Bass T, Sabaté M. Vessel shrinkage as a sign of atherosclerosis progression in type 2 diabetes: a serial intravascular ultrasound analysis. Diabetes. 2009;58:209-14.

23. Kedhi E, Kennedy MW, Maehara A, Lansky AJ, McAndrew TC, Marso SP, De Bruyne B, Serruys PW, Stone GW. Impact of TCFA on Unanticipated Ischemic Events in Medically Treated Diabetes Mellitus: Insights From the PROSPECT Study. JACC Cardiovasc Imaging. 2017;10:451-8. 